\title{
Inactivation du gène de la neurofibromatose (NF1) chez la souris
}

La neurofibromatose de type 1 (NF1) est une maladie autosomique dominante atteignant environ 1 personne sur 3500 . Elle se manifeste avant tout par des neurofibromes et des taches cutanées café au lait. Il existe cependant un risque de tumeurs malignes incluant gliomes, neurosarcomes, phéochromocytomes et leucémies myéloïdes. Son gène siège en $17 q 11.2$ et la protéine, dite "neurofibromine", longue de 2818 acides aminés, est un membre de la famille GAP (GTPase activating proteins) des protéines régulatrices Ras. Le gène a été cloné en $1990(\mathrm{~m} / \mathrm{s}$ $n^{\circ} 8$, vol. $\left.6, p .816\right)$. Il est considéré comme un suppresseur de tumeurs. Pour aider à comprendre le mécanisme de fonctionnement d'un tel gène, un modèle animal était hautement souhaitable. Deux équipes américaines, celle de Brannan et al.[1] (Frederick, MD, New Haven, C.T et Cincinnati, OH) et celle de Jacks et al. [2] (Boston, MA), en ont entrepris la réalisation. Ils ont utilisé la méthode désormais classique d'invalidation, insérant une cassette neo dans un gène de cellules embryonnaires, ES, puis plaçant ces cellules dans des blastocystes pour obtenir des souris hétérozygotes et, enfin, par croisement, des homozygotes.

Dans les deux groupes, les animaux homozygotes ne sont pas viables. Ils meurent entre les jours 12 et 14 de la vie embryonnaire. La principale anomalie est cardiaque, les deux gros vaisseaux se branchant sur le seul ventricule droit. Une telle observation était inattendue, car les atteintes cardiaques ne font pas partie du tableau habituel de la NFl humaine. Par ailleurs, un seul des deux groupes [1] a observé d'autres anomalies, telles qu'un retard général de développement et une hypertrophie des ganglions sympathiques dérivés de la crête neurale. Les animaux hétérozygotes ont été particulièrement étudiés par Jacks et al. [2], pendant 27 mois; pour ceux de Brannan et al. [1], aucune anomalie n'était perceptible à 10 mois. Tout d'abord, manquaient les deux principaux symptômes de la maladie humaine, neurofibromes et troubles de la pigmentation cutanée. Il ne s'agit donc pas d'un modèle idéal de la NFl humaine. En revanche, et en accord avec l'hypothèse d'un suppresseur de tumeur, le taux et la vitesse d'apparition de tumeurs malignes sont accrus. Dans l'ensemble, ces tumeurs sont les mêmes que celles qui atteignent les souris normales, mais plus tard pour ces dernières, la mutation semblant accélérer le développement des tumeurs auxquelles les souris sont susceptibles. Cependant une fréquence relative d'affections malignes, connues dans la NFl, se manifeste sous forme de phéochromocytomes (rares chez la souris normale) et de leucémies myéloïdes. Deux autres aspects de ce travail peuvent être soulignés. Les auteurs ont voulu mettre à l'épreuve le modèle à deux coups de Kuudson, et donc chercher la présence ou l'absence de l'allèle normal de neurofibromine dans les tumeurs : la moitié environ des tissus testés (mais la totalité des huit phéochromocytomes et des sept leucémies myéloïdes) avaient perdu l'allèle normal, reconnaissable en Southern blot. Il n'a pas été contrôlé si, lorsque l'allèle normal était présent, il avait pu subir une mutation.
L'autre discussion intéressante porte sur la nature de la mutation expérimentale. Dans l'article de Brannan et al., le messager n'était présent qu'à l'état de traces. Au contraire, dans celui de Jacks et al., le transcrit muté est en quantité normale et le messager apparaît stable. Il est reconnaissable car de taille plus grande. La raison de cette contradiction n'est pas immédiatement apparente, car l'intégration du gène neo se fait dans le même exon dans les deux séries d'expériences. Aucune trace de protéine neurofibromine n'a pu être détectée chez les homozygotes par immunologie, avec des anticorps dirigés contre des épitopes différents, et chez les hétérozygotes on trouve la moitié de la dose normale. On peut donc considérer le mutant comme nul.

Les souris hétérozygotes ne constituent donc pas un modèle satisfaisant de NFl ; on peut néanmoins en tirer une conclusion intéressante, la confirmation du rôle de suppresseur de tumeur de la neurofibromine. Il n'est, par ailleurs, pas nouveau que l'invalidation de gènes murins ne reproduise pas correctement, ou même pas du tout, le tableau de la maladie humaine provoquée par une lésion moléculaire analogue. De fait, le meilleur modèle clinique de NFl chez la souris a été obtenu par une lignée transgénique portant le gène HTLV-1-tat [3]. Il serait intéressant de croiser cette lignée avec celle déficiente en neurofibromine.

Un mystère à élucider est celui des lésions cardiaques des homozygotes déficients. Il existe dans la crête neurale des oiseaux des cellules qui contribuent à la formation des gros vaisseaux du coeur [4] ; chez la sou-

. 
ris, on observe une forte élévation de la neurofibromine vers 12 jours [5]; enfin, une forme de NFl humaine, dite "de Watson", comporte une anomalie cardiaque sous forme d'une sténose de la valvule pulmonaire [6]. L'observation d'une malformation cardiaque chez des souris homozygotes pour l'absence de neurofibromine met donc bien en évidence les relations entre crête neurale et développement des vaisseaux cardiaques.

Ces résultats posent encore la ques- tion de savoir si une NFl humaine homozygote serait viable. Nous n'avons pas connaissance de la description de tels cas.

\section{J.C.D.}

1. Brannan CI, Perkins AS, Vogel KS, et al. Targeted disruption of the neurofibromatosis type-1 gene leads to developmental abnormalities in heart and various neural crest-derived tissues. Genes Der $1994 ; 8$ : 1019-29.

2. Jacks T, Shih TS, Schmiu EM, Bronson RT, Bernards A, Weinberg RA. Tumour predisposition in mice heterozygous for a targeted mutation in NFI. Nature Genet $1994 ; 7: 353-61$.

3. Hinrichs SH, Nerenberg M, Reynolds RK,
Khoury (;, Jay (;A. A transgenic mouse model for human neurofibromatosis. Science $1987 ; 237$ 1.340-3.

4. Kirby MI., Gale TF, Stewart DE. Neural crest cells contribute to aorticopulmonary septation. Science 1983 ; 220 : 1059-61

5. Huynh DP, Nechiporuk T, Pulst SM. Differential expression and tissue distribution of type 1 and type II neurofibromins during mouse fetal development. Dev Biol 1994 ; 161: 538-51.

6. Tassabehji M, Strachan T, Sharland M, Colley A, Donnai D, Harris R, Thakker N. Tandem duplication within a neurofibromatosis type I (NF1) gene exon in a family with features of Watson syndrome and Noonan syndrome. Am J Hum Genet $1993 ; 53: 90-5$.
Quand l'image est malade... et le malade bien portant, il y a problème ! Le raffinement des techniques d'imagerie, notamment par résonance magnétique nucléaire, permet des avancées considérables dans le diagnostic de nombreuses affections neurologiques. Il existe toutefois, et tous les radiologues le savent, une variabilité biologique et une tolérance individuelle dont l'étendue est souvent surprenante. L'image en elle-même n'est donc qu'un élément qu'il faut dans la plupart des cas confronter soigneusement à la clinique pour tirer des conclusions. Las! même la plus belle association anatomo-clinique peut être trompeuse comme le suggère une étude coordonnée par Michael Brant-Zawadski (Newport, Californie USA) sur l'IRM de la colonne vertébrale chez des individus ne présentant pas de lombalgie [1]. Sur 98 sujets, sains cliniquement, 62 présentaient des anomalies radiologiques soit près de deux sur trois! Sachant que des douleurs lombaires plus ou moins sévères font partie des plaintes de plus d'un adulte sur deux, il est évident que l'on retrouve dans la plupart des cas une remarquable cohérence anatomo-clinique lorsque l'on pratique les examens chez les patients cliniquement atteints... ne serait-ce que dans deux tiers des cas ! Le problème est que cette cohérence peut n'être qu'apparen- image radiologique de lésion chronique parfaitement tolérée et un symptôme clinique peut-être dû à une autre atteinte. Sachant que l'effet d'annonce peut en lui-même être considérable sur les conséquences d'un symptôme douloureux, on ne peut qu'être poussé à la prudence. Il ne faut sûrement pas jeter au feu l'IRM lombaire mais sa prescription devrait probablement être limitée aux cas qui, cliniquement, semblent appeler un traitement chirurgical ou un diagnostic vital [2].

[1. Jensen MC, et al. $N$ Engl J Med 1994 ; 331 : 69-73.]

[2. Deyo RA. N Engl I Med 1994 ; 331 : 115-6.]

Homologie du gène eyeless de la drosophile avec les homéogènes $P a x-6$ murin et humain, aux mutations responsables des phénotypes Small eye de la souris et Aniridia de l'homme. Les gènes $P a x$ codent pour des facteurs de transcription qui se lient à des séquences spécifiques de l'ADN et jouent un rôle majeur dans le développement embryonnaire, en particulier du système nerveux [1]. On connaît des mutants de trois des neuf gènes $P a x$ identifiés : la mutation undulated de Paxl chez la souris qui entraîne chez les homozygotes un développement anormal des vertèbres et du sternum $\left(\mathrm{m} / \mathrm{s} n^{\circ} 2\right.$, vol. 5, p. 125), la mutation de $\operatorname{Pax} 3$, responsable chez l'homme du syndrome de Waardenburg qui associe une surdité, des troubles de la pigmentation, une hétérochromie de l'iris et une prédisposition au spina bifida, syndrome voisin de celui décrit chez la souris sous le nom de splotch $\left(\mathrm{m} / \mathrm{s} n^{\circ} 4\right.$, vol. 8 , p. 393), la mutation de Pax6 caractérisée chez la souris par une microphtalmie à l'état hétérozygote et une absence totale d'œil et de cavité nasale (létale) à l'état homozygote, et, chez l'homme, par une aniridie $\left(\mathrm{m} / \mathrm{s} n^{\circ} 2\right.$, vol. $\left.8, \mathrm{p} .181\right)$. Une équipe suisse de Bâle vient de montrer l'homologie du gène Pax6 de la drosophile (dont la mutation est connue sous le nom de eyeless) avec les gènes Pax6 déjà décrits dans les autres espèces [2]. Elle montre que le gène Pax6 est très conservé, l'identité des séquences d'acides aminés des protéines codées étant supérieure à $90 \%$ entre drosophile, souris, rat, poisson-zèbre et homme. Pax6 est impliqué dans le contrôle génétique de la morphologie de l'œil dans toutes ces espèces, des mammifères aux insectes, ce qui conduit à reconsidérer l'idée traditionnelle que les yeux des vertébrés et les yeux très complexes des insectes se seraient développés indépendamment.

[1. Babinet C. médecine/sciences $1993 ; 9$ : 87-9.]

[2. Quiring R, et al. Science 1994 ; $265: 785-9$. 
Fécondation d'ovocytes murins par des spermatides. Le plus jeune gamète mâle ayant achevé une méiose complète est le spermatide rond qui doit subir de nombreuses modifications pour être transformé en spermatozoïde mobile et fécondant. Cependant, des résultats récents, commentés par le Comité consultatif national d'éthique dans un avis daté du mois de juillet [1], ont montré que, chez. les humains, l'ovocyte pouvait être fécondé par injection intracytoplasmique de spermatozoïdes immobiles. Une équipe japonaise (Tokyo) et améri caine (Honolulu, Hawaii) [2] démontre maintenant que, chez la souris, l'électror fusion de spermatides ronds avec des ovocytes permet, assez fréquemment, d'obtenir une fécondation aboutissant à ın embryon se développant normalement. En pathologie humaine, il existe certains cas, quoique relativement rares, où des hommes stériles azoospermiques ont une spermatogenèse bloquée après le stade des spermatides. L'application de la technique décrite chez la souris à ces cas pourrait permettre de proposer à ces hommes d'être des pères biologiques. Espérons que l'expérimentation préalable sur des modèles animaux, puisqu'elle s'avère ici possible, permettra d'accumuler le maximum de renseignements fondant non seulement l'efficacité mais aussi l'innocuité de cette méthode.

[1. Comité national consultatif d'éthique (France). médecine/sciences 1994 ; 10 : 925-7.]

[2. Ogura A, et al. Proc Natl Acad Sci USA 1994 ; 91 : 7460-2.]

\footnotetext{
Association des transporteurs de peptides avec le complexe majeur d'histocompatibilité de classe I. Les molécules de classe I du complexe majeur d'histocompatibilité (CMH-I) lient, dans le réticulum endoplasmique (RE), des peptides provenant de la dégradation des protéines cytosoliques et les
}

présentent aux lymphocytes T CD8 cytotoxiques. Ces peptides, produits dans le cytosol par les protéasomes, sont transloqués dans le RE par des transporteurs membranaires composés de deux sous-unités (TAP-1 et TAP-2) [1]. A moins d'être liés au CMH-I, les ligands peptidiques ne sont pas détectés dans la cellule, et sont probablement rapidement dégradés dans le cytosol et/ou le RE. L'association des peptides avec le $\mathrm{CMH}$ doit pourtant être efficace malgré des conditions défavorables telles que la dégradation, la dilution après passage dans le RE, la liaison aux molécules chaperons... En fait, ces problèmes sont minimisés, car, comme le montrent deux rapports récents $[2,3]$, le CMH-I, vide de peptides, est associé aux transporteurs TAP. Il peut être chargé de peptides in vitro, ce qui provoque sa séparation d'avec les complexes TAP. In vivo, la formation de dimères CMH-I (chaîne lourde- $\beta 2$ microglobuline) serait favorisée par la calnexine et suivie de leur association aux transporteurs TAP. Cette association faciliterait la liaison des peptides transloqués par les TAP au CMH-I, peut-être en augmentant localement la concentration des ligands et des récepteurs.

[1. Bahram S. médecine/sciences 1993; 9 : 1204-13.]

[2. Suh WK, et al. Science 1994 ; $264: 1322-6$.

[3. Ortmann B, et al. Nature 1994 : $368: 864-7$.

- Syndrome de Miller-Dieker et PAF-hydrolase. Le syndrome de Miller-Dieker est une malformation cérébrale marquée par le caractère lisse de la surface cérébrale (lissencéphalie). Il parait dû à une insuffisance de migration des neurones cérébraux. On trouve chez les malades une délétion en 17pl3.3, incluant le gène d'une protéine LISl, qui a été rendue responsable de la maladie $\left(\mathrm{m} / \mathrm{s} n^{\circ} 11\right.$, vol. 9, p. 1124). Des auteurs japonais viennent de faire une découverte surprenante: on connaît, depuis que Jacques Benveniste et al l'ont décrit en $1972\left(\mathrm{~m} / \mathrm{s} n^{\circ} 9\right.$, vol. 3, p. 506), un composé appelé PAF-acéther* dont le rôle est important ; il est inactivé par désacétylation, sous l'action d'une enzyme très répandue, la PAF acétylhydrolase. Celle-ci possède trois sous-unités, de 29,30 et 45 kDa [1]. On sait que la sous-unité $29 \mathrm{~K}$ porte l'activité catalytique et on ignore la fonction des autres. Hatteri et al [2] ont cloné l'ADNc de la $45 \mathrm{~K}$ de cerveau de bœuf et ont constaté que, sur 410 acides aminés, 407 étaient identiques à ceux de L.ISl humaine. Il est donc très probable que LISl et la $45 \mathrm{~K}$ de la PAF acétylhydrolase sont identiques. On sait que L.ISl possède la structure répétitive des $\beta$-transducines, mais celles-ci sont loin d'exhiber une homogénéité de fonction. Ce résultat imprévu pose plus de questions qu'il n'en résout. Certes le PAF et son récepteur sont présents dans le système nerveux et le PAF est peut-être un neurotransmetteur; la sous-unité $45 \mathrm{~K}$ possède vraisemblablement un rôle régulateur. Mais on ne voit pas actuellement comment l'absence d'une sous-unité régulatrice suffirait pour empêcher des neurones de migrer correctement. Nous n'avons, par ailleurs, pas trouvé de données concernant la fonction de la PAF acétylhydrolase chez les malades, qui, il est vrai, sont des hétérozygotes.

[1. Hattori M, et al. J Biol Chem 1993; 268: 13748-53.]

[2. Hattori M, et al. Nature 1994 ; $370: 216-8$.]

[3. Hanahan D. Annu Rev Biochem $1986 ; 55: 483-509$.]

* Ie PAF-acéther est un dévivé du glvcérol, acétylé sur le carbone central (sn2) et pontant sur le C:3 (sn3) un groupe phosphonglcholine [3].

* Le PAlf-acéther est un dérivé du glycérol, acétylé sur
le carbone central (sn2) el portant sur le C $3(\operatorname{sn} 3)$ un
groupe phosplonglcholine [3].

